\title{
Ten-Year Results of Laparoscopic Sleeve Gastrectomy: Retrospective Matched Comparison with Laparoscopic Adjustable Gastric Banding-Is There a Significant Difference in Long Term?
}

\author{
Mario Musella ${ }^{1} \cdot$ Giovanna Berardi $^{1} \cdot$ Nunzio Velotti $^{1} \cdot$ Vincenzo Schiavone $^{1} \cdot$ Antonio Vitiello $^{1}$ (D
}

Received: 4 May 2021 / Revised: 15 September 2021 / Accepted: 22 September 2021 / Published online: 3 October 2021

(c) The Author(s) 2021

\begin{abstract}
Background The laparoscopic sleeve gastrectomy (LSG) is the most common bariatric procedure performed worldwide while the laparoscopic adjustable gastric banding (LAGB) has been almost abandoned. Aim of this study was to retrospectively assess 10-year outcomes of LSG through a matched comparison with LAGB.

Materials and Methods Retrospective search of prospectively maintained database of our university was carried out to find all patients that underwent LSG before December 2010. Each subject with LSG was matched one-to-one with a patient that had undergone LAGB in the same period with correspondent preoperative age, BMI, and sex.

Results A total of 76 patients underwent LSG before 2010 and were all included in this study; a matched group of 76 out of 178 LAGB patients with 10-year follow-up was retrieved from our database. Comparison between the two groups showed better outcomes after LSG at 1 and 5 years but weight loss was comparable with the LAGB group at 10 years (\%TWL $22.2 \pm 13$ vs $21.2 \pm 16.1 ; p=0.89)$. No significant difference was found in conversion/removal rate $(15.8 \%$ vs $18.4 \% ; p=0.67)$. Conclusion LSG is an effective stand-alone bariatric procedure with better outcomes than LAGB in medium term, but results are comparable at 10 years. Subjects undergoing LSG should be informed that conversion to RYGB or OAGB may be necessary to achieve further weight loss or to treat reflux.
\end{abstract}

Keywords Sleeve gastrectomy $\cdot$ Laparoscopic adjustable gastric banding $\cdot$ Long-term results $\cdot$ Bariatric surgery

\section{Introduction}

Key points

- LSG shows superiority over LAGB in short and medium term.

- Weight loss and conversion rate are comparable at 10 years.

- Subjects undergoing LSG should be informed that conversion to RYGB or OAGB may be necessary in long term.

\author{
Antonio Vitiello \\ antoniovitiello_@hotmail.it \\ Mario Musella \\ mario.musella@unina.it \\ Giovanna Berardi \\ giovannaberardi88@gmail.com \\ Nunzio Velotti \\ nunzio.velotti@gmail.com \\ Vincenzo Schiavone \\ vincenzoschiavone92@gmail.com \\ 1 Advanced Biomedical Sciences Department, Naples \\ "Federico II" University, AOU "Federico II", Via S. Pansini \\ 5, 80131 Naples, Italy
}

History of restrictive bariatric surgery goes back to the vertical banded gastroplasty (VBG) [1], which was described by Mason in 1982. This intervention was later modified by MacLean et al. [2], who suggested to divide the gastric pouch from the remnant stomach. Aim of this modification was to prevent recanalization between the pouch and the fundus in order to avoid weight regain. Long-term weight loss ranged from 42 to $69 \% \mathrm{EWL}$ (excess weight loss percent) [3-8], but laparoscopic approach and revisional surgery were challenging tasks after VBG. In the early 2000s, the laparoscopic adjustable gastric band (LAGB) replaced VBG as the most common restrictive procedure. Indeed, LAGB accounted for $24.4 \%$ of all weight-loss interventions in 2003 and for $42.3 \%$ in 2008 [9, 10]. However, several long-term reports demonstrated a non-response rate up to $40-50 \%$ [11-13] and LAGB started to be abandoned. 
In 2014, the percentage of bandings performed worldwide decreased to $7.4 \%$, also due to the success of the laparoscopic sleeve gastrectomy (LSG) [14].

Eventually in 2016, LSG overcame the Roux-en-Y gastric bypass (RYGB) as the most common intervention worldwide [15] accounting for $46 \%$ of all bariatric interventions in 2018 [16].

However, outcomes of LSG have been recently questioned by several studies, whose results have shown a worrisome rate of postoperative GERD (gastroesophageal reflux disease) $[17,18]$. Some articles have also described intestinal metaplasia (Barrett's disease) after LSG due to the chronic exposure of the lower esophagus to reflux [19, 20].

Since laparoscopic restrictive surgery has been widely performed at our university, some years ago we faced the wave of patients with LAGB coming back to clinic asking for removal or conversion to other interventions. Presently, we are experiencing the same unpleasant situation with subjects who had LSG more than 5 years ago. Therefore, longterm duration of weight loss after the sleeve gastrectomy has been questioned.

Aim of this study was to retrospectively assess 10 -year outcomes of LSG through a matched comparison with LAGB with special regard to rates of success $(\% \mathrm{EWL}>50)$, non-response (\%EWL $<25)$, weight regain, and conversion.

\section{Methods}

Retrospective search of prospectively maintained database of our university was carried out to find all consecutive patients who underwent LSG at our department before December 2010. Inclusion criteria were age between 18 and 60 years, BMI $>40 \mathrm{~kg} / \mathrm{m}^{2}$ or $>35$ with an obesity-related disease. Subjects with a previous history of bariatric or abdominal surgery were excluded. Each subject who underwent LSG was matched one-to-one with a patient treated with LAGB in the same period with correspondent preoperative BMI, age, and sex ( \pm 1 year age for a given BMI unit).

Collected data at baseline were sex, age, body mass index (BMI), obesity-related diseases, and GERD. Weight loss was analyzed at 1,5 , and 10 years of follow-up. Removal/conversion rate and GERD improvement/worsening were evaluated at 10 years.

Ethics Committee of our institution approved the study and informed consent was obtained from each participant.

\section{Surgical Technique}

Surgical techniques for both procedures have been described in detail elsewhere [21,22], but a brief description is reported below for completeness of the article.
For LAGB, a total number of 4 trocars $(2 \times 5 \mathrm{~mm}$; $1 \times 10 \mathrm{~mm}, 1 \times 15 \mathrm{~mm}$ ) were placed. The operation started with the dissection of the gastrophrenic ligament and the opening of the pars flaccida of the small omentum. After the creation of a retrogastric tunnel, the band was drawn along this path and closed. Two gastro-gastric sero-serous nonabsorbable sutures were passed between the gastric fundus and the gastric pouch above the band.

For LSG, a five-trocar approach $(3 \times 12 \mathrm{~mm}, 2 \times 5 \mathrm{~mm})$ was used. The gastrectomy started $4-6 \mathrm{~cm}$ from the pylorus over a 38-40 French bougie. Staple line reinforcements or oversewing is not routinely used at our institution.

\section{Preoperative Evaluation and Follow-up}

All patients were preoperatively evaluated by a multidisciplinary team consisting of endocrinologists, psychiatrists, dieticians, and surgeons. Liquid diet was started on postoperative day (POD) 1 for LAGB and POD 3 for LSG and discharge was planned the day after. Pureed foods were allowed after postoperative day 15 and normal diet after 30 days. Follow-up appointments were routinely planned at 1, 3, 6, and 12 months. After the first year, visits were planned every 6-12 months. Band regulations were decided on the base of symptoms and weight.

\section{Weight Loss}

Weight loss was calculated as percentage of excess weight loss (\%EWL), total weight loss percent (\%TWL), and excess body mass index loss percent (\%EBMIL) using the following formulas:

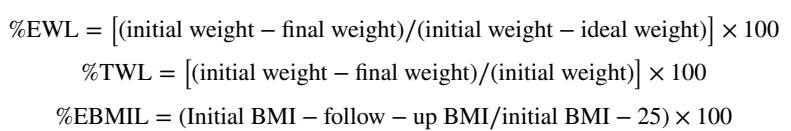

Success at 10 years was defined as $\% E W L \geq 50$; nonresponse was set as $\% \mathrm{EWL}<25$ [23], while weight regain was set as $\% \mathrm{EWL}<50$ at 10 years for a patient who had previously achieved \%EWL $>50$. Percentage of patients with $\mathrm{BMI}>35,35<\mathrm{BMI}<30$, and $\mathrm{BMI}<30 \mathrm{~kg} / \mathrm{m}^{2}$ was also calculated according to Biron's classification of bariatric results [24].

\section{Remission from Obesity-Related Disease}

Remission of type 2 diabetes (T2DM) was considered as a value of glycated hemoglobin A1c (HbA1C) $<6.5 \%$ off antidiabetic medications [25]. Hypertension (HTN) remission 
was defined as blood pressure $<140 / 90$ off antihypertensive medication [26].

\section{GERD}

At our center, GERD symptoms are routinely investigated in all patients during preoperative and postoperative appointments. According to the Lyon Consensus Conference [27] criteria, de novo GERD was clinically diagnosed in case of new onset heartburn and regurgitation after surgery.

\section{Complications}

Early postoperative complications (bleeding, perforation, staple line leak, stenosis, untreatable vomiting) in the first 30 days and late complications (slippage, erosion/migration, port/tube infection, late staple line leak, and stenosis) were recorded.

\section{Statistical Analysis}

Data are expressed as mean $\pm \mathrm{SD}$. Paired $t$-test was used to compare continuous variables as appropriate, while categorical data were compared using the chi-square test and Fisher's exact test. Significant $p$ value was set below 0.05 .

\section{Results}

A total of 152 (52 males/100 females) patients were included in this study; 76 patients underwent LSG before 2010 and were all included in this study; and a matched group of 76 out of 178 LAGB patients with 10-year follow-up was retrieved from our database. Baseline demographics are reported in Table 1.

\section{Total Number of LAGB with 10-Year Follow-up}

An overall number of 225 patients had undergone LAGB before 2010, but 47 (21\%) subjects were loss during

Table 1 Baseline characteristics. BMI, body mass index; GERD, gastroesophageal reflux disease; $L S G$, laparoscopic sleeve gastrectomy; $L A G B$, laparoscopic adjustable gastric band

\begin{tabular}{llll}
\hline Characteristic & $L S G(n=76)$ & $L A G B(n=76)$ & $p$ value \\
\hline Male gender & $26(34.2 \%)$ & $26(34.2 \%)$ & 1 \\
Age (years) & $38.1 \pm 9$ & $38.5 \pm 11.7$ & 0.38 \\
Initial BMI $\left(\mathrm{kg} / \mathrm{m}^{2}\right)$ & $45.1 \pm 4.8$ & $45.2 \pm 4.8$ & 0.16 \\
Diabetics & $6(7.9 \%)$ & $1(1.3 \%)$ & 0.11 \\
Hypertension & $12(15.8 \%)$ & $6(7.9 \%)$ & 0.13 \\
Preoperative GERD & $4(5.2 \%)$ & $3(3.9 \%)$ & 1 \\
\hline
\end{tabular}

follow-up. Therefore, data of 178 (79\%) patients are available in our database.

Overall band removal rate at 10 years was $19.1 \%(n=34)$; $6(3.4 \%)$ were removed for complications and $28(15.7 \%)$ for insufficient weight loss.

\section{Follow-up, Complication, and Removal/Conversion Rates}

Due to conversion or removal, follow-up at 1, 5, and 10 years was $100 \%, 100 \%$, and $92.1 \%$ in the LSG group and $100 \%$, $94.7 \%$, and $81.6 \%$ in the LAGB group.

No major postoperative complication occurred in the two groups.

In the LAGB group, port/tube complications (leak or disconnection leading to infections) occurred in 14/76 (18.4\%); drainage, replacement, and repositioning were performed when appropriate [28, 29]. Removal was necessary for 3 $(3.9 \%)$ subjects after the fifth year for severe dysphagia.

Four LAGBs were removed in the first 5 years due to insufficient weight loss (IWL, $25<\%$ EWL $<50$ ) or nonresponse; later, 7 additional patients underwent removal and conversion to LSG $(n=4)$ or OAGB $(n=3)$ for IWL.

In the LSG group, no patient required conversion in the first 5 years, but afterwards 6 patients were converted to one anastomosis gastric bypass/mini-bypass (OAGB) to achieve further weight loss. At the tenth year, 1 subject underwent reLSG and 3 were submitted to OAGB for weight regain, while other 2 (2.6\%) were converted to RYGB for severe reflux.

Rates and causes of removal/conversion are summarized in Table 2.

\section{Weight Loss}

Comparison between the two groups showed better outcomes after LSG at 1 and 5 years but weight loss did not result significantly different from the LAGB group at 10 years (Table 3; Fig. 1).

Analysis of BMI according to Biron's classification showed that half of patients in both groups had BMI $>35$ at 10 years (Fig. 2 and 3 ).

Table 2 Comparison of rates and causes of conversion/removal. $I W L$, insufficient weight loss; GERD, gastroesophageal reflux disease; $L S G$, laparoscopic sleeve gastrectomy; $L A G B$, laparoscopic adjustable gastric band

\begin{tabular}{|c|c|c|c|}
\hline $\begin{array}{l}\text { Reason for conversion/ } \\
\text { removal }\end{array}$ & $L S G(n=76)$ & $L A G B(n=76)$ & $p$ value \\
\hline $\begin{array}{l}\text { Complications (GERD or } \\
\text { band complications) }\end{array}$ & $2(2.6 \%)$ & $3(3.9 \%)$ & 1 \\
\hline IWL or non-response & $10(13.2 \%)$ & $11(14.5 \%)$ & 1 \\
\hline Conversion/removal rate & $12 / 76(15.8 \%)$ & $14 / 76(18.4 \%)$ & 0.67 \\
\hline
\end{tabular}


Table 3 Comparison of weight loss in the two groups. BMI, body mass index; \%EWL, percentage of excess weight loss; \%EBMIL, excess body mass index loss percent; $\% T W L$, total weight loss percent; $L S G$, laparoscopic sleeve gastrectomy; $L A G B$, laparoscopic adjustable gastric band

\begin{tabular}{lllc}
\hline & $L S G(n=76)$ & $L A G B(n=76)$ & $p$ value \\
\hline BMI 1 year & $35.1 \pm 5.4$ & $37.7 \pm 5.2$ & $<0.0001$ \\
BMI 5 years & $33.9 \pm 5.3$ & $37.5 \pm 6.8$ & 0.0003 \\
BMI 10 years & $34.7 \pm 5.4$ & $35.6 \pm 7.4$ & 0.98 \\
\%EWL 1 year & $51.1 \pm 26.2$ & $38.7 \pm 19.1$ & 0.0004 \\
\% EWL 5 years & $55.7 \pm 27.2$ & $40.1 \pm 29.3$ & 0.0005 \\
\% EWL 10 years & $50.1 \pm 30.5$ & $47.3 \pm 35.2$ & 0.88 \\
\%TWL 1 year & $22.2 \pm 10.7$ & $16.8 \pm 8.4$ & 0.0002 \\
\%TWL 5 years & $30.7 \pm 15.5$ & $22.4 \pm 17.1$ & 0.0006 \\
\%TWL 10 years & $22.2 \pm 13$ & $21.2 \pm 16.1$ & 0.89 \\
\%EBMIL 1 year & $50.5 \pm 26.5$ & $37.4 \pm 19.6$ & 0.0002 \\
\%EBMIL 5 years & $55.1 \pm 27.7$ & $38.7 \pm 30.1$ & 0.0004 \\
\%EBMIL 10 years & $49.5 \pm 30.9$ & $46 \pm 36$ & 0.89 \\
EWL > 50\% at 10 years & $34 / 70(48.6 \%)$ & $30 / 62(48.4 \%)$ & 0.98 \\
EWL < 25\% at 10 years & $14 / 70(20 \%)$ & $20 / 62(32.3 \%)$ & 0.11 \\
Weight regain at 10 years & $4 / 70(5.7 \%)$ & $10 / 62(16.1 \%)$ & 0.05 \\
\hline
\end{tabular}

\section{Remission from Obesity-Related Disease and GERD}

After 10 years, all patients with preoperative diabetes were still taking drugs; rate of remission from hypertension was $58 \%$ after LSG and $16.7 \%$ after LAGB.

Eighteen cases of de novo GERD occurred after LSG and 4 after LAGB; symptoms did not improve for the only patient who suffered from preoperative reflux (Table 4).

\section{Discussion}

Currently VBG is not included in the guidelines of bariatric surgery while LSG is the most common intervention performed worldwide and LAGB has been almost abandoned.

Main reason of the decline of LAGB was the rate of nonresponse or weight regain, as reported in series with medium [30] and long-term follow-up [31, 32]. A systematic review of studies with 10-year follow-up and a total of 9706 patients [33] showed an average \%EWL of 49 at 10 years with a $30 \%$ removal rate. Nevertheless, recent studies reported that, considering its reversibility and safety, LAGB could still be proposed for selected patients [34].

Conversely, LSG was initially introduced by Marceau et al. [35] as first part of the duodenal switch operation in order to preserve vagal innervation and pyloric function. Subsequently, Regan et al. [36] proposed a staged procedure also for gastric bypass, mostly to overcome the challenge of laparoscopic surgery in patients with BMI $>60 \mathrm{~kg} / \mathrm{m}^{2}$. Since postoperative outcomes demonstrated low morbidity and satisfactory weight loss, LSG achieved the status of a bariatric intervention [37].

Short-term studies (1-3 years) on LSG have reported an excellent outcome in terms of excess weight loss (\%EWL), which is comparable to values after RYGB [38]. Mid-term reports (5-7 years) have shown less successful results, since weight recidivism rate was estimated to be $27.8 \%$ with a range of 14 to $37 \%$ [39-41]; the SM-BOSS [42] study showed that BMI loss peaked at 2 years after SG $(74.7 \%)$ but decreased by the end of the fifth year to $61.1 \%$. In a previous retrospective comparison [40], patients with LSG achieved better weight loss than LAGB at 5 years, but comparable remission from comorbidities. Medium-term studies also
Fig. 1 Weight loss comparison between the two groups

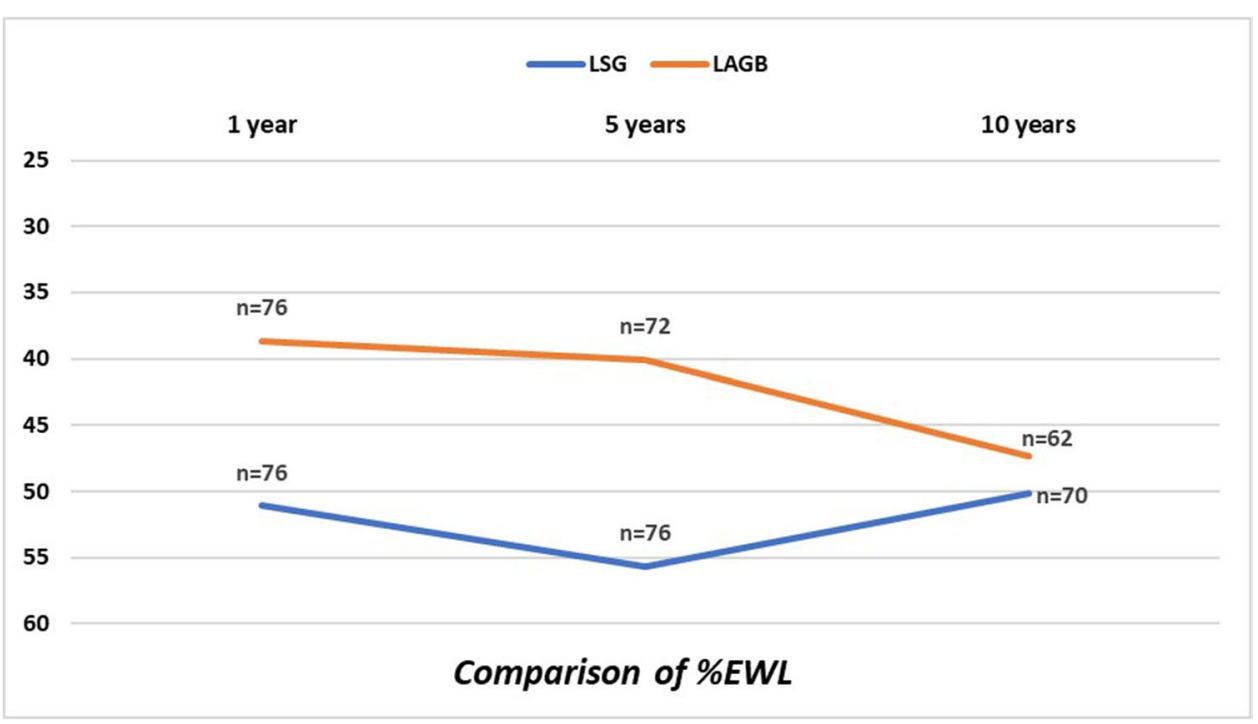


Fig. 2 LSG group BMI analysis

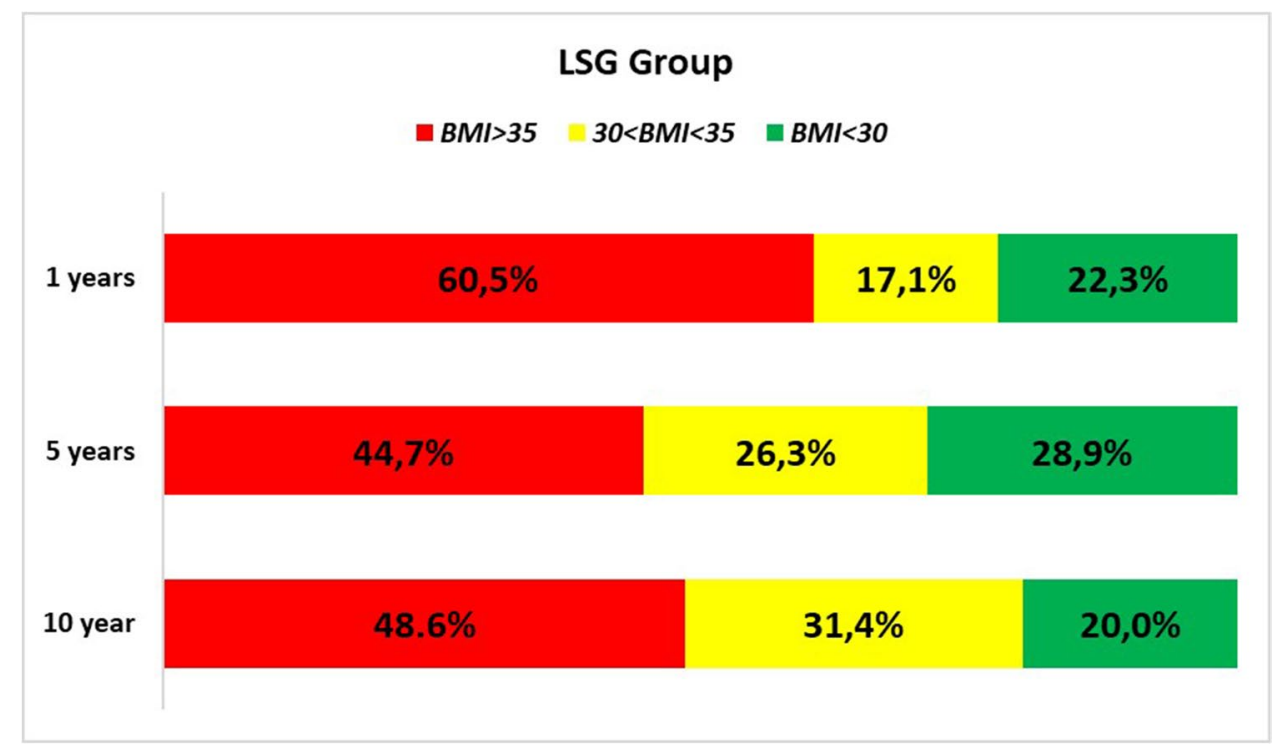

Fig. 3 LAGB group BMI analysis

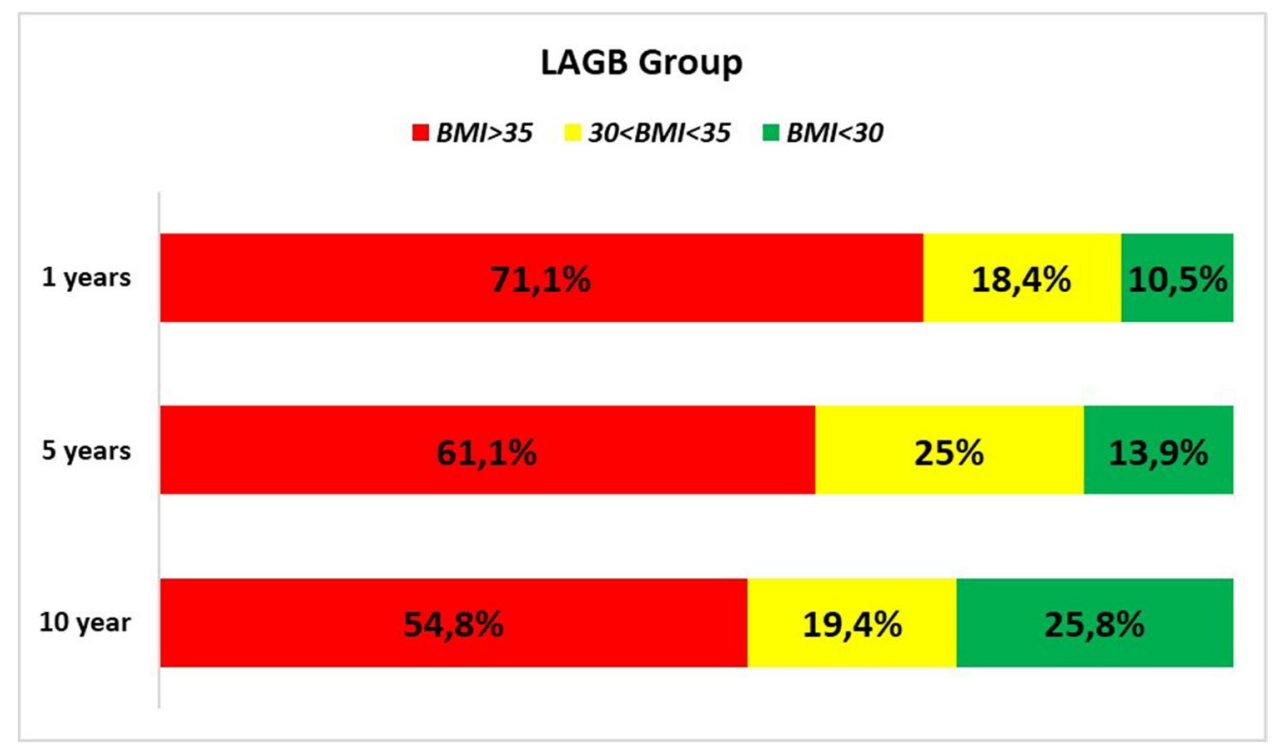

Table 4 Remission from comorbidities and GERD (gastroesophageal reflux disease). $L S G$, laparoscopic sleeve gastrectomy; $L A G B$, laparoscopic adjustable gastric band

\begin{tabular}{llll}
\hline & $L S G$ & $L A G B$ & $p$ value \\
\hline Remission from hypertension & $7 / 12(51.4 \%)$ & $1 / 6(16.7 \%)$ & 0.15 \\
Remission from diabetes & $0 / 6(0 \%)$ & $0 / 1(0 \%)$ & 1 \\
Remission from GERD & $0 / 4(0 \%)$ & $0 / 3(0 \%)$ & 1 \\
De novo GERD & $18 / 70(25.7 \%)$ & $4 / 62(6.5 \%)$ & 0.004 \\
\hline
\end{tabular}

demonstrated a rate of remission from diabetes and hypertension of almost 70\% [43] after LSG.

Surprisingly, despite LSG has been widely performed in the last 20 years, there is a lack of long-term studies
(8-10 years). Felsenreich et al. [44] reported 54\% EWL after a mean follow-up of 10.8 years with sample size of 53 patients, while Arman et al. [45] found 62.5\%EWL in 110 patients.

Very recently an article with results beyond 10 years has been published: even if LSG provided a long-term \%EBMIL ranging from 51 to $54 \%$, high incidence of insufficient weight loss and de novo reflux was observed; conversion to other interventions was necessary in $19.2 \%$ of cases.

Even if some studies reported improvement of symptoms after SG [46, 47], most articles show a worsening of preoperative reflux and de novo GERD [48]. Undoubtedly, the main reason for new onset heartburn is the increased intragastric pressure (IGP) caused by a sleeved stomach, resulting in a decreased compliance. Also, technical mistakes could 
lead to a stenotic or twisted sleeve, which causes regurgitation of acid content into the esophagus.

Braghetto and Csendes [49] first reported an incidence of $1.2 \%$ of BE at 1 year after LSG; lately other authors reported a rate of $15-17 \%$ after a longer follow-up (5 to 10 years) [20]. A recent systematic review showed a rate of de-novo GERD of 20\% [50] after LSG, while a meta-analysis found that the increase of postoperative GERD was $19 \%$ and de novo reflux occurred in 23\% [51] of patients. Our study shows an important incidence of clinical GERD after LSG, being one fourth of patients diagnosed with heartburn and regurgitation at 10 years.

Even if the outcomes of the present study match the results of the abovementioned literature, comparison between LAGB and LSG showed interesting results at 10 years.

In short and medium term, superiority of LSG is clear, but the two procedures show a similar outcome at 10 years. Success of LSG is mainly due to the massive results achievable in the first postoperative years, when weight loss is definitively better and probably comparable to RYGB. However, as said before, after the fifth year a progressive weight regain begins which may be irreversible in the long term.

Remarkably, the curve of weight loss after LAGB continuously slopes down, while the curve after LSG starts to rise after the fifth year. Explanation of this different trend probably relies in the different history of the two interventions. In the last 10 years, LAGB has been considered not very successful, then patients with initial signs of IWL or non-response have rapidly undergone removal or conversion. Subsequently, there is an early drop-out of subjects with bad results from the LAGB group; therefore, only patients with better results are included in the long-term follow-up.

On the other hand, weight regain was not expected after LSG and conversion to another procedure was often delayed. Indeed, half of conversions after the sleeve occurred at the tenth year. Probably, the risk of regaining kilos after LSG is higher for those subjects with IWL. Indeed, we found that only $5.7 \%$ of patients with $\% \mathrm{EWL}>50$ before year 5 failed to maintain this result in long term. In this view, it is interesting that conversion/removal rate was comparable at 10 years and that the main reason for revisional surgery was IWL or non-response in both groups.

It is undeniable that all surgical units are recently facing a wave of patients with LSG requiring conversion, as happened years ago with subjects who had undergone LAGB. This trend will probably continue in the next years, since $48.6 \%$ of sleeved subjects still have indication for bariatric surgery (BMI $>35 \mathrm{~kg} / \mathrm{m}^{2}$ in long term).

Nevertheless, it must be underlined that almost half of LSG patients have conserved a successful mean weight loss $(\mathrm{EWL}>50 \%)$ at 10 years, proving that the sleeve gastrectomy deserves to be considered a stand-alone procedure.
In our bariatric center, patients with morbid obesity and multiple comorbidities are usually submitted to malabsorptive procedures; therefore, numbers are too small to draw serious conclusions on comorbidities remission, but improvement really happened only after LSG.

\section{Strength and Limitations}

The present study reports outcomes of a matched retrospective study with acceptable sample size considering the longterm follow-up.

However, comparison could be biased by initial technique for LSG, which was fashioned less tightly over larger bougies. Currently, the sleeve operation is performed over a 38 French tube starting $4 \mathrm{~cm}$ from the pylorus; some authors [52] have stated that a radical antrectomy could improve and accelerate weight loss.

Also, GERD assessment was only clinical, and although international validated standard questionnaires were used, and data from instrumental investigations were often unavailable.

\section{Conclusion}

LSG is an effective bariatric procedure in short and medium term with clear superiority over LAGB. However, after 10 years, weight loss and conversion rate were comparable between the two procedures and $15.8 \%$ of LSG patients required reoperation. Long-term RCT are needed to better understand the rate for conversion.

Patients with morbid obesity should be adequately counseled to LAGB or LSG. Those subjects unwilling to undergo an irreversible, even if more effective, procedure could be submitted to LAGB; patients selected for LSG should be informed that conversion to RYGB [53] or OAGB [54] may be necessary to achieve further weight loss or to treat reflux.

Acknowledgements Authors want to thank Prof. Maurizio Taglialatela of University Federico II of Naples for his help with the editing of the manuscript.

Funding Open access funding provided by Universita degli Studi di Napoli Federico II within the CRUI-CARE Agreement.

\section{Declarations}

Ethics Approval All procedures performed in studies involving human participants were in accordance with the ethical standards of the institutional and/or national research committee and with the 1964 Helsinki Declaration and its later amendments or comparable ethical standards. 
Consent to Participate Informed consent was obtained from all individual participants included in the study.

Conflict of Interest The authors declare no competing interests.

Open Access This article is licensed under a Creative Commons Attribution 4.0 International License, which permits use, sharing, adaptation, distribution and reproduction in any medium or format, as long as you give appropriate credit to the original author(s) and the source, provide a link to the Creative Commons licence, and indicate if changes were made. The images or other third party material in this article are included in the article's Creative Commons licence, unless indicated otherwise in a credit line to the material. If material is not included in the article's Creative Commons licence and your intended use is not permitted by statutory regulation or exceeds the permitted use, you will need to obtain permission directly from the copyright holder. To view a copy of this licence, visit http://creativecommons.org/licenses/by/4.0/.

\section{References}

1. Mason EE. Vertical banded gastroplasty for obesity. Arch Surg. 1982;117:701-6.

2. MacLean LD, Rhode BM, Forse RA. A gastroplasty that avoids stapling in continuity. Surgery. 1993;113:380-8.

3. Baltasar A, Bou R, Arlandis F, et al. Vertical banded gastroplasty at more than 5 years. Obes Surg. 1998;8(1):29-34. https://doi.org/10.1381/096089298765555015.

4. Bekheit M, Katri K, Salam WN, et al. Rejecting the demise of vertical-banded gastroplasty: a long-term single-institute experience. Obes Surg. 2013;23:1604-10.

5. del Amo DA, Diez MM, Guedea ME, et al. Vertical banded gastroplasty: is it a durable operation for morbid obesity? Obes Surg. 2004;14:536-8.

6. Lin YH, Lee WJ, Ser KH, et al. 15-Year follow-up of verticalbanded gastroplasty: comparison with other restrictive procedures. Surg Endosc. 2016;30:489-94.

7. Schouten R, Wiryasaputra DC, van Dielen FM, et al. Long-term results of bariatric restrictive procedures: a prospective study. Obes Surg. 2010;20:1617-26.

8. Scozzari G, Toppino M, Famiglietti F, et al. 10-Year follow up of laparoscopic vertical banded gastroplasty: good results inselected patients. Ann Surg. 2010;252:831-9.

9. Buchwald H, Oien DM. Metabolic/bariatric surgery worldwide 2008. Obes Surg. 2009;19(12):1605-11.

10. Buchwald H, Williams SE. Bariatric surgery worldwide 2003. Obes Surg. 2004;14(9):1157-64.

11. Suter M, Calmes JM, Paroz A, et al. A 10-years experience with laparoscopic gastric banding for morbid obesity: high long-term complication and failure rates. Obes Surg. 2006;16:829-35.

12. Topart P, Becouarn G, Ritz P. One-year weight loss after primary or revisional Roux-en-Y gastric bypass for failed adjustable gastric banding. Surg Obes Relat Dis. 2009;5:459-62.

13. De Maria EJ, Sugerman HJ, Meador JG, et al. High failure rate after laparoscopic adjustable silicone gastric banding for treatment of morbid obesity. Ann Surg. 2001;223:809-18.

14. Angrisani L, Santonicola A, Iovino P, et al. Erratum to: bariatric surgery and endoluminal procedures: IFSO worldwide survey 2014. OBES SURG. 2017;27:2290-2. https://doi.org/10.1007/ s11695-017-2773-8.

15. Angrisani L, Santonicola A, Iovino P, et al. IFSO worldwide survey 2016: primary, endoluminal, and revisional procedures. Obes Surg. 2018;28(12):3783-94.
16. Welbourn R, Hollyman M, Kinsman R, et al. Bariatric surgery worldwide: baseline demographic description and one-year outcomes from the fourth IFSO global registry report 2018. Obes Surg. 2019;29(3):782-95.

17. Stenard F, Iannelli A. Laparoscopic sleeve gastrectomy and gastroesophageal reflux. World J Gastroenterol. 2015;21(36):10348-57.

18. Melissas J, Braghetto I, Molina JC, et al. Gastroesophageal reflux disease and sleeve gastrectomy. Obes Surg. 2015;25(12):2430-5

19. Genco A, Soricelli E, Casella G, et al. Gastroesophageal reflux disease and Barrett's esophagus after laparoscopic sleeve gastrectomy: a possible, underestimated long-term complication. Surg Obes Relat Dis. 2017;13(4):568-74.

20. Felsenreich DM, Kefurt R, Schermann M, et al. Reflux, sleeve dilation, and Barrett's esophagus after laparoscopic sleeve gastrectomy: long-term follow-up. Obes Surg. 2017;27(12):3092-101. https://doi.org/10.1007/s11695-017-2748-9.

21. Musella M, Milone M, Gaudioso D, Bianco P, Palumbo R, Galloro G, Bellini M, Milone F. A decade of bariatric surgery. What have we learned? Outcome in 520 patients from a single institution. Int J Surg. 2014;12(Suppl 1):S183-8. https://doi.org/ 10.1016/j.ijsu.2014.05.012.

22. Vitiello A, Berardi G, Velotti N, De Palma GD, Musella M. Is there an indication left for gastric band? A single center experience on 178 patients with a follow-up of 10 years. Updates Surg. 2021;73(2):657-62. https://doi.org/10.1007/s13304-020-00858-8.

23. Brolin R, Kenler HA, Gorman RC, et al. The dilemma of outcome assessment after operations for morbid obesity. Surgery. 1989;105:337-46.

24. Biron S, Hould FS, Lebel S, Marceau S, Lescelleur O, Simard S, Marceau P. Twenty years of biliopancreatic diversion: what is the goal of the surgery? Obes Surg. 2004;14(2):160-4. https://doi.org/ 10.1381/096089204322857492.

25. American Diabetes Association. Diagnosis and classification of diabetes mellitus. Diabetes Care. 2010;33(Suppl 1):S62-9.

26. National Institute for Health and Clinical excellence. https://www. nice.org.uk/guidance/ng136/chapter/recommendations. NICE guideline [NG136] Published date: August 2019

27. Gyawali CP, Kahrilas PJ, Savarino E, Zerbib F, Mion F, Smout AJPM, Vaezi M, Sifrim D, Fox MR, Vela MF, Tutuian R, Tack J, Bredenoord AJ, Pandolfino J, Roman S. Modern diagnosis of GERD: the Lyon Consensus. Gut. 2018;67(7):1351-62.

28. Tog CH, Halliday J, Khor Y, et al. Evolving pattern of laparoscopic gastric band access port complications. OBES SURG. 2012;22:863-5. https://doi.org/10.1007/s11695-011-0567-y.

29. Keidar A, Carmon E, Szold A, et al. Port complications following laparoscopic adjustable gastric banding for morbid obesity. Obes Surg. 2005 Mar;15(3):361-5. https://doi.org/10.1381/09608 92053576604. PMID: 15826470. Jul;67(7):1351-1362. https:// doi.org/10.1136/gutjnl-2017-314722. Epub 2018 Feb 3. PMID: 29437910; PMCID: PMC6031267

30. O'Brien PE, McPhail T, Chaston TB, et al. Systematic review of medium-term weight loss after bariatric operations. Obes Surg. 2006;16(8):1032-40.

31. Froylich D, Abramovich-Segal T, Pascal G, et al. Long-term (over 10 years) retrospective follow-up of laparoscopic adjustable gastric banding [published correction appears in Obes Surg. 2017 Dec 1;:]. Obes Surg. 2018. 28(4):976-980

32. Naef M, Mouton WG, Naef U, et al. Graft survival and complications after laparoscopic gastric banding for morbid obesity-lessons learned from a 12-year experience. Obes Surg. 2010;20(9):1206-14.

33. Shen X, Zhang X, Bi J, et al. Long-term complications requiring reoperations after laparoscopic adjustable gastric banding: a systematic review. Surg Obes Relat Dis. 2015;11(4):956-64. 
34. Arapis K, Tammaro P, Parenti LR, et al. Long-term results after laparoscopic adjustable gastric banding for morbid obesity: 18-year follow-up in a single university unit. Obes Surg. 2017;27(3):630-40.

35. Marceau P, Biron S, Bourque RA, et al. Biliopancreatic diversion with a new type of gastrectomy. Obes Surg. 1993;3(1):29-35. https://doi.org/10.1381/096089293765559728.

36. Regan JP, Inabnet WB, Gagner M, et al. Early experience with two-stage laparoscopic Roux-en-Y gastric bypass as an alternative in the super-super obese patient. Obes Surg. 2003;13(6):861-4. https://doi.org/10.1381/096089203322618669.

37. Gumbs AA, Gagner M, Dakin G, et al. Sleeve gastrectomy for morbid obesity. Obes Surg. 2007;17(7):962-9. https://doi.org/10. 1007/s11695-007-9151-x.

38. Leyba JL, Aulestia SN, Llopis SN. Laparoscopic Roux-enY gastric bypass versus laparoscopic sleeve gastrectomy for the treatment of morbid obesity. A prospective study of 117 patients. Obes Surg. 2011;21(2):212-6. https://doi.org/10.1007/ s11695-010-0279-8.

39. Clapp B, Wynn M, Martyn C, et al. Long term (7 or more years) outcomes of the sleeve gastrectomy: a meta-analysis. Surg Obes Relat Dis. 2018;14(6):741-7. https://doi.org/10.1016/j.soard. 2018.02.027.

40. Jain M, Tantia O, Goyal G, et al. LSG vs MGB-OAGB: 5-year follow-up data and comparative outcome of the two procedures over long term-results of a randomised control trial. Obes Surg. 2021;31(3):1223-32. https://doi.org/10.1007/ s11695-020-05119-6.

41. Peterli R, Borbély Y, Kern B, et al. Early results of the Swiss Multicentre Bypass or Sleeve Study (SM-BOSS): a prospective randomized trial comparing laparoscopic sleeve gastrectomy and Roux-en-Y gastric bypass. Ann Surg. 2013;258(5):690-4. https:// doi.org/10.1097/SLA.0b013e3182a67426 (discussion 695).

42. Vitiello A, Pilone V, Ferraro L, et al. Is the sleeve gastrectomy always a better procedure? Five-year results from a retrospective matched case-control study. Obes Surg. 2018;28(8):2333-8.

43. Eid GM, Brethauer S, Mattar SG, et al. Laparoscopic sleeve gastrectomy for super obese patients: forty-eight percent excess weight loss after 6 to 8 years with 93\% follow-up. Ann Surg. 2012;256:262-5.

44. Felsenreich DM, Langer FB, Kefurt R, et al. Weight loss, weight regain, and conversions to Roux-en-Y gastric bypass: 10-year results of laparoscopic sleeve gastrectomy. Surg Obes Relat Dis. 2016;12:1655-62.
45. Arman GA, Himpens J, Dhaenens J, et al. Long-term (11+years) outcomes in weight, patient satisfaction, comorbidities, and gastroesophageal reflux treatment after laparoscopic sleeve gastrectomy. Surg Obes Relat Dis. 2016;12:1778-86.

46. Rebecchi F, Allaix ME, Giaccone C, et al. Gastroesophageal reflux disease and laparoscopic sleeve gastrectomy: a physiopathologic evaluation. Ann Surg. 2014;260:909-14.

47. Pilone V, Tramontano S, Renzulli M, et al. Gastroesophageal reflux after sleeve gastrectomy: new onset and effect on symptoms on a prospective evaluation. Obes Surg. 2019;29(11):3638-45. https://doi.org/10.1007/s11695-019-04046-5.

48. Iannelli A, Sans A. Martini $F$ et al Hiatal hernia, GERD, and sleeve gastrectomy: a complex interplay. Obes Surg. 2016;26(10):2485-7.

49. Braghetto I, Csendes A. Prevalence of Barrett's esophagus in bariatric patients undergoing sleeve gastrectomy. Obes Surg. 2016;26(4):710-4. https://doi.org/10.1007/s11695-015-1574-1.

50. Oor JE, Roks DJ, Ünlü Ç, et al. Laparoscopic sleeve gastrectomy and gastroesophageal reflux disease: a systematic review and meta-analysis. Am J Surg. 2016;211(1):250-67.

51. Yeung KTD, Penney N, Ashrafian L, et al. Does sleeve gastrectomy expose the distal esophagus to severe reflux?: a systematic review and meta-analysis. Ann Surg. 2020;271(2):257-65. https:// doi.org/10.1097/SLA.0000000000003275.

52. Pizza F, D’Antonio D, Lucido FS, et al. Does antrum size matter in sleeve gastrectomy? A prospective randomized study. Surg Endosc. 2021;35:3524-32. https://doi.org/10.1007/ s00464-020-07811-1.

53. Frezza EE, Ikramuddin S, Gourash W, et al. Symptomatic improvement in gastroesophageal reflux disease (GERD) following laparoscopic roux-en-Y gastric bypass. Surg Endosc. 2002;16(7):1027-31.

54. Musella M, Bruni V, Greco F, et al. Conversion from laparoscopic adjustable gastric banding (LAGB) and laparoscopic sleeve gastrectomy (LSG) to one anastomosis gastric bypass (OAGB): preliminary data from a multicenter retrospective study. Surg Obes Relat Dis. 2019;15(8):1332-9.

Publisher's Note Springer Nature remains neutral with regard to jurisdictional claims in published maps and institutional affiliations. 